\title{
Short Communication Induction of lymphangiogenesis in and around axillary lymph node metastases of patients with breast cancer
}

\author{
GG Van den Eynden', I Van der Auwera', SJ Van Laere', V Huygelen', CG Colpaert', P van Dam', LY Dirix', \\ PB Vermeulen*,I and EA Van Marck' \\ 'Translational Cancer Research Group Antwerp(Lab Pathology University Antwerp/University Hospital Antwerp, Edegem; Oncology Center, General \\ Hospital St-Augustinus, Wilrijk), Antwerp, Belgium
}

Lymph node (LN) status is the most important prognostic factor for patients with breast cancer. The presence and the extent of axillary LN metastases reflect the probability that the cancerous process has spread through the body and both are strongly correlated with the development of distant metastases and with shortened disease-free and overall survival. Lymph node metastases are more than passive tumour deposits. Metastatic tumour sites are capable of inducing a vascular stroma and can actively contribute to tumour progression and to further metastatic spread. To what extent processes involved in progression of primary tumours, such as angiogenesis and lymphangiogenesis, contribute to progression of secondary sites is largely unknown. Reports have suggested differences between primary tumours and secondary sites and between different secondary sites. Whereas primary breast tumours grow angiogenesis dependently, we demonstrated that $90 \%$ of breast cancer liver metastases grow according to an angiogenesis-independent replacement pattern (Stessels et al, 2004). The growth of breast cancer LN metastases, on the contrary, was angiogenesis dependent and angiogenesis and hypoxia in the metastases were correlated with angiogenesis and hypoxia in the primary tumours (Van den Eynden et al, 2005). Guidi et al (2000) demonstrated that the presence of vascular hot spots in LN metastases, but not in the primary breast tumours was associated with decreased survival.

In the present study, we compared the expression of the lymphatic endothelium-specific markers Prox-1, LYVE-1 and podoplanin in metastatically involved and uninvolved LNs of patients with breast cancer. Prox-1 and LYVE-1 are, respectively, a transcription factor and a hyaluronan receptor that show

*Correspondence: Dr PB Vermeulen, Department of Pathology, AZ St-Augustinus, Oosteveldlaan 24, B26I 0 Wilrijk, Belgium;

E-mail: peter.vermeulen@gvagroup.be

Received 27 April 2006; revised 27 September 2006; accepted 27 September 2006; published online 31 October 2006 specificity for lymphatic endothelial cells. D2-40 was originally described as a selective monoclonal antibody to a $M_{\mathrm{r}} 40000$ O-linked sialoglycoprotein that reacts with a fixation-resistant epitope in lymphatic endothelium (Kahn and Marks, 2002). Recently, the D2-40 antibody has been shown to specifically recognise podoplanin, a glomerular podocyte membrane protein (Schacht et al, 2005) and has been shown to be a very sensitive and specific marker for lymphatic endothelium in most tissues (Evangelou et al, 2005) and especially in breast cancer (Van der Auwera et al, 2005). We investigated the presence and extent of lymphangiogenesis in LN metastases of breast cancer using the podoplanin antibody.

\section{MATERIALS AND METHODS}

\section{Patients and samples}

One hundred and ten patients with operable breast cancer were included in this study, 49 patients with LN-negative and 61 patients with LN-positive breast cancer. Clinico-pathological features are compared between both study groups in Table 1 , using the UICC TNM system. Formalin-fixed paraffin-embedded tissue blocks of one metastatically involved and - if available - one uninvolved LN of patients with LN-positive breast cancer and one metastatically uninvolved LN of patients with LN-negative breast cancer were selected for immunohistochemical examination. Of six LN-positive patients, no uninvolved LN was available.

\section{Expression of lymphatic endothelium-specific and vascular markers}

Immunohistochemical stainings for the lymphatic endotheliumspecific markers podoplanin (clone D2-40, Dako, Glöstrup, Denmark), Prox-1 (polyclonal, Reliatech, Braunschweig, Germany) and LYVE-1 (polyclonal, Reliatech) and for the panendothelial 
Table I Comparison of clinico-pathological variables of the primary tumour in $\mathrm{NO}$ and $\mathrm{N}+$ patients included

\begin{tabular}{|c|c|c|c|}
\hline & No $(n=49)$ & $N+(n=61)$ & $P$-value \\
\hline Mean age (range) & $60.9(34.3-85.4)$ & $59.6(25.3-84.4)$ & 0.63 \\
\hline Histology & & & 0.81 \\
\hline IDA & 40 & 48 & \\
\hline ILA & 6 & 10 & \\
\hline Other & 3 & 3 & \\
\hline T status & & & 0.004 \\
\hline TI & 28 & 15 & \\
\hline $\mathrm{T} 2$ & 18 & 37 & \\
\hline T3 & 2 & 5 & \\
\hline T4 & 1 & 4 & \\
\hline$N$ status & & & NA \\
\hline No & 49 & 0 & \\
\hline $\mathrm{NI}$ & 0 & 27 & \\
\hline N2 & 0 & 20 & \\
\hline N3 & 0 & 14 & \\
\hline$E R$ & & & 0.78 \\
\hline Negative & 14 & 16 & \\
\hline Positive & 35 & 45 & \\
\hline$P R$ & & & 0.26 \\
\hline Negative & 18 & 29 & \\
\hline Positive & 31 & 32 & \\
\hline Her-2/neu & & & 0.6 \\
\hline Negative & 42 & 50 & \\
\hline Positive & 7 & 11 & \\
\hline
\end{tabular}

$\mathrm{ER}=$ oestrogen receptor, IDA = infiltrating ductal adenocarcinoma; ILA = infiltrating lobular adenocarcinoma; $\mathrm{NO}=$ lymph node negative, $\mathrm{N}+=$ lymph node positive; $\mathrm{PR}=$ progesteron receptor. Age, histological type, $\mathrm{T}$ and $\mathrm{N}$ status, ER and PR status, HER-2/neu oncoprotein status were recorded by review of the pathology files. In N+ patients, tumours were larger than in NO patients.

markers CD34 (clone QBEnd10, Dako) and CD31 (clone JC70A, Dako) were performed on serial sections of a subset $(n=20)$ of metastatically involved and uninvolved LNs. A detailed description of the protocol of these stainings has been published before (Van der Auwera et al, 2005) (Figure 1).

\section{Presence of lymph vessels and lymphangiogenesis}

An immunohistochemical staining for podoplanin (clone D2-40, Dako) and an immunohistochemical double staining with antipodoplanin (clone D2-40, Dako) and anti-Ki-67 antibodies (clone MIB-1, Dako) were performed on sections of all included cases. The presence of lymph vessels (LVs) was assessed intranodally (within the LN capsule) of metastatically involved and uninvolved LNs, and, if LVs were present, intranodal lymphatic endothelial cell proliferation fraction (LECP\%) was assessed by counting Ki-67 positive and negative lymphatic endothelial cells. If perinodal LVs were present, perinodal LECP\% was also assessed. Only cases with $>10$ lymphatic endothelial cells were included for statistical analysis.

\section{Statistical analysis}

Statistical analyses were performed with the SPSS 13.0 software package. A $P$-value $<0.05$ was considered statistically significant. Correlations between categorical variables (e.g. presence of LVs, metastatical involvement) were analysed using a $\chi^{2}$ test. LECP\% in different groups was compared with a Kruskal-Wallis nonparametric test.

\section{RESULTS}

Results of the comparison of the expression of the different vascular markers in and around metastatically uninvolved LNs of patients with breast cancer are shown in Table 2. Although the littoral cells of the marginal and trabecular sinuses were very focally and faintly immunoreactive for podoplanin, no intranodal podoplanin immunoreactive vascular structures were demonstrated except in trabecular septa or areas of lipomatosis and fibrosis (Figure 1A-F). In contrast, in most metastatically involved LNs vascular structures staining for podoplanin were observed. These structures were also positive for Prox-1, CD31 and CD34. They were only faintly LYVE-1 positive or did not show LYVE-1 immunoreactivity (Figure $1 \mathrm{G}-\mathrm{K}$ ).

We then analysed the presence of LVs in the total study population, using podoplanin as a lymphatic marker. Lymph vessels were only seen in 26 out of $104(25.0 \%)$ uninvolved LNs. In 22 of these $26(84.6 \%)$ LNs, LVs were seen in areas of lipomatosis or fibrosis or in trabecular septa. There was no difference in the presence of LVs in uninvolved LNs of patients with LN-negative and LN-positive breast cancer $(P=0.91)$. In contrast, LVs were demonstrated in 52 of $61(85.2 \%)$ metastatically involved LNs $(P<0.001)$ (Table 3$)$. These LVs were often localised at the metastasis/LN interface or in the LN parenchyma close to the metastasis.

Figure 2 shows Box and Whisker's plots comparing intranodal (Figure 2A) and perinodal (Figure 2B) LECP\% in all groups. The median intranodal LECP\% was $6.0 \%(n=42)$ in metastatically involved LNs compared to $1.6 \%(n=11)$ in uninvolved LNs of LN-negative and $0.0 \%$ in uninvolved LNs $(n=9)$ of LN-positive patients $(P<0.001)$. The median perinodal LECP\%s were $2.9,0.0$ and $0.0 \%$ in metastatically involved LNs $(n=25)$, uninvolved LNs from LN-positive patients $(n=37)$ and in uninvolved LNs from LN-negative patients $(n=20)$, respectively $(P<0.001)$.

\section{DISCUSSION}

To the best of our knowledge, this is the first report demonstrating the presence of lymphangiogenesis in secondary sites of human cancer in general and in LN metastases of breast cancer in particular. First, different lymphatic endothelial-specific markers were compared. As LYVE-1 reactivity of LVs close to LN metastases decreases, as LYVE-1 reactivity of macrophages and intravascular proteins in both blood and LVs hampers interpretation (see Figure 1) and as Prox-1 is a nuclear antigen, we preferred to use podoplanin (clone D2-40) to study the presence of LVs and lymphangiogenesis in the total study population. In a previous study, we have shown that although LYVE-1 expression on LVs located at the tumour periphery was strong, no or weak immunoreactivity was found in intratumoral LVs and that podoplanin is the best marker to visualise LVs in and around primary breast tumours (Van der Auwera et al, 2005).

In primary human tumours, the contribution of lymphangiogenesis $v s$ lymphatic cooption and the functionality of tumour lymphatics are still controversial. Nevertheless, LVs containing proliferating nuclei have been observed in breast cancer (Van der Auwera et al, 2005), endometrial cancer (Koukourakis et al, 2005), head and neck cancer (Beasley et al, 2002) and melanoma (Dadras et al, 2003; Straume et al, 2003). As LVs were only found in a minority of uninvolved axillary LNs, the increase of the presence of LVs in metastatically involved LNs is likely to be due to lymphangiogenesis. An increased LECP\% was indeed found in and around metastatically involved LNs. Although misinterpretation of nuclei from proliferating tumour cells penetrating the vessel wall or underlying the LV as endothelial cell nuclei might lead to falsely elevated LECP\%, careful interpretation by experienced investigators can largely reduce this bias. Tumour cell nuclei and endothelial cell nuclei mostly differ from shape and the use of 

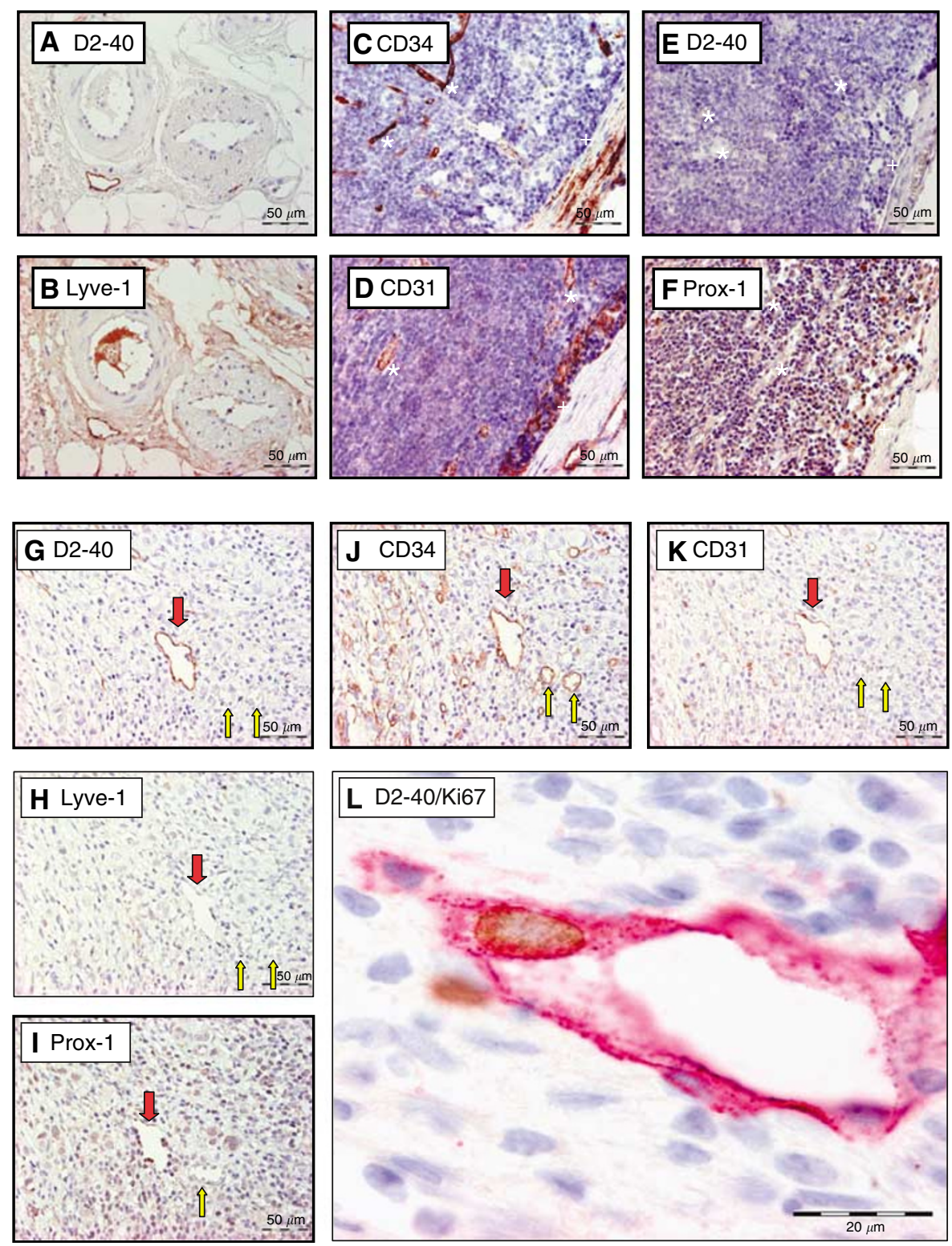

Figure I Overview of the immunohistochemical stains used on serial sections of a metastatically uninvolved (perinodal fat: $\mathbf{A}-\mathbf{B}$; LN: C-F) and involved LN (G-L). In uninvolved LNs, high endothelial venules (*) show CD34 (C) and CD3I (D) reactivity. Littoral cells (+) are positive for CD3I (D) and ProxI (F). In metastatically involved LNs, podoplanin-positive LVs (red arrow) are demonstrated (G). Blood vessels (yellow arrow) are podoplanin negative (G). The LVs show CD34 (J), CD3I (K) and Prox-I (I) reactivity. Although in perinodal fat (B) LVs are strongly positive for LYVE-I, the LVs in metastatically involved LNs are only faintly positive for LYVE-I $(\mathbf{H})$. Furthermore, a proliferating lymphatic endothelial cell is shown at the border of a LN metastasis $(\mathbf{L})$ (LN: lymph node; LV: lymph vessel).

microscopy at different focal planes further reduces this bias. The methodology adopted in this study to measure ECP\% is widely accepted in the angiogenesis field (Vermeulen et al, 2002) and has recently been introduced in the lymphangiogenesis field (Dadras et al, 2003; Beasley et al, 2002; Straume et al, 2003; Koukourakis et al, 2005; Van der Auwera et al, 2005). The median intranodal LECP $\%$ of $6.0 \%$ suggests that lymphangiogenesis in metastatically involved LNs is higher than in primary breast tumours: we previously demonstrated that LECP\% in primary breast tumours was $1.83 \%$ (Van der Auwera et al, 2005).

Our results corroborate the concept of LN lymphangiogenesis, which was recently introduced and shown to be involved in the recruitment of dendritic cells to inflamed LNs (Angeli et al, 2006). The authors demonstrated that the lymphangiogenic response in the LNs was particularly localised in the subcapsular space and around B-cell follicles from where LVs penetrated into the cortex.
Whether the outgrowth of new LVs in metastatically involved axillary LNs originates from the subcapsular marginal sinus or from LVs in areas of lipomatosis or in fibrous septa remains to be elucidated.

Different hypotheses could be raised about the role of LN lymphangiogenesis in metastatically involved LNs. On the one hand, LN lymphangiogenesis might be part of the immunological reaction against the tumour cells and on the other hand be involved in tumour progression and metastases, as in primary tumours. Therefore, LN lymphangiogenesis, as LN angiogenesis, might contribute to the metastatic spread of breast cancer. In a transgenic mouse model of skin cancer, Hirakawa et al (2005) demonstrated that the induction of lymphangiogenesis by vascular endothelial growth factor $\mathrm{A}$ is involved in tumour progression. They furthermore showed that VEGF-A also induces lymphangiogenesis in the sentinel $\mathrm{LN}$ and that lymphangiogenesis is induced 
Table 2 Expression of different vascular markers in metastatically uninvolved LNs of patients with breast cancer

\begin{tabular}{|c|c|c|c|c|c|}
\hline & CD3I & CD34 & Podoplanin & Prox-I & LYVE-I $^{a}$ \\
\hline $\begin{array}{l}\text { High endothelial } \\
\text { venules }\end{array}$ & + & + & - & - & - \\
\hline $\begin{array}{l}\text { Littoral cells (lining } \\
\text { subcapsular and } \\
\text { trabecular sinuses) }\end{array}$ & + & - & $\begin{array}{l}\text { Very focally } \\
\text { and faintly }\end{array}$ & + & Faintly \\
\hline \multicolumn{6}{|c|}{ Fibrous capsule and trabecular fibrous septa } \\
\hline BV & $+{ }^{b}$ & $++^{b}$ & - & - & - \\
\hline LV & $+^{\mathrm{b}}$ & $+^{\mathrm{b}}$ & + & + & + \\
\hline \multicolumn{6}{|c|}{ Fibrous and lipomatous areas } \\
\hline BV & $t^{b}$ & $\mathrm{t}^{\mathrm{b}}$ & - & - & - \\
\hline LV & $+^{\mathrm{b}}$ & $++^{b}$ & + & + & + \\
\hline \multicolumn{6}{|l|}{ Perinodal fat } \\
\hline BV & $++^{\mathrm{b}}$ & $++^{\mathrm{b}}$ & - & - & - \\
\hline LV & $+^{\mathrm{b}}$ & $++^{\mathrm{b}}$ & + & + & + \\
\hline
\end{tabular}

Table 3 Comparison of the presence of LVs between metastatically involved and uninvolved LNs

\begin{tabular}{lrc}
\hline & \multicolumn{3}{c}{ Presence of LVs } \\
\cline { 2 - 3 } & No & Yes \\
\hline Uninvolved LN & & \\
NO breast cancer & 37 & 12 \\
N+ breast cancer & 41 & 14 \\
Metastatically involved LN & 9 & 52 \\
\hline
\end{tabular}

$L N=$ lymph node; $L V=$ lymph vessel, $N 0=L N$ negative; $N+=L N$ positive. Uninvolved $\mathrm{LNs}$ are divided in $\mathrm{LNs}$ from $\mathrm{NO}$ and $\mathrm{N}+$ patients with breast cancer. $P$-value (metastatically involved vs uninvolved $L N s$ ) $<0.001$. P-value (uninvolved LNs No vs $\mathrm{N}+)=0.91$.

before metastasising (Hirakawa et al, 2005). Based on our data, it is difficult to investigate the mutual contribution of local and remote mechanisms to the induction of lymphangiogenesis in LNs of patient with breast cancer. However, the difference in LECP\% in uninvolved LNs of patients with $\mathrm{N} 0$ vs $\mathrm{N}+$ breast cancer is intriguing in this context.

\section{ACKNOWLEDGEMENTS}

G Van den Eynden is a research assistant of the Fund for Scientific Research Flanders. S Van Laere is supported by a Research Grant

\section{REFERENCES}

Angeli V, Ginhoux F, Llodrà J, Quemeneur L, Frenette P, Skobe M, Jessberger R, Merad M, Randolph G (2006) B cell-driven lymphangiogenesis in inflamed lymph nodes enhances dendritic cell mobilization. Immunity 24: 203

Beasley N, Prevo R, Banerji S, Leek R, Moore J, Van Trappen P, Cox G, Harris AL, Jackson DG (2002) Intratumoral lymphangiogenesis and lymph node metastasis in head and neck cancer. Cancer Res 62: 1315

Dadras SS, Bertoncini J, Brown L, Muzikansky A, Jackson D, Ellwanger U, Garbe C, Mihm M, Detmar M (2003) Tumor lymphangiogenesis: a novel prognostic indicator for cutaneous melanoma metastasis and survival. Am J Pathol 162: 1951
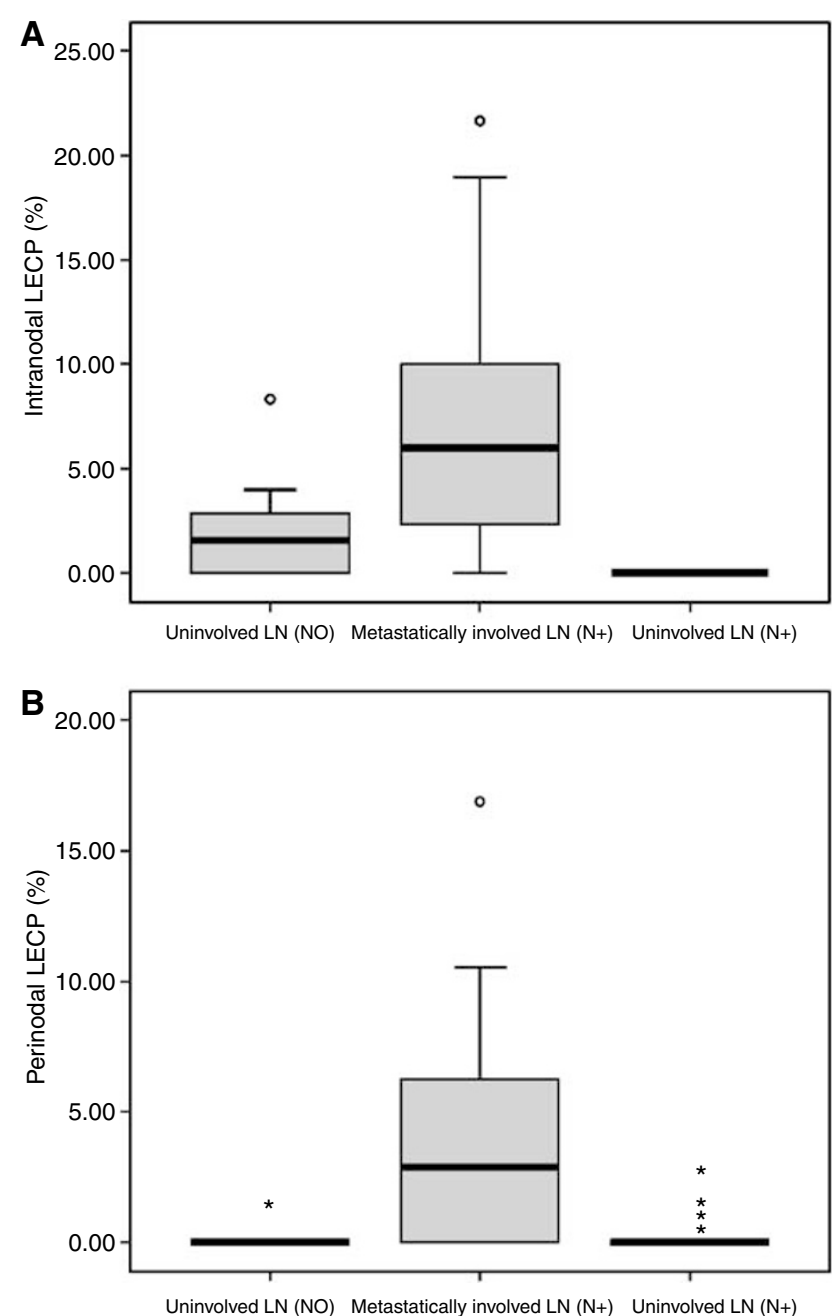

Figure 2 Comparison of intranodal (A) and perinodal (B) LECP\% between metastatically involved and uninvolved axillary $\mathrm{LNs}$ in patients with $\mathrm{LN}$-negative and $\mathrm{LN}$-positive breast cancer. Both intra- $(P<0.00 \mathrm{I})$ and perinodal $(P<0.001)$ LECP\% is significantly increased in metastatically involved LNs compared to uninvolved LNs (LECP\%: lymphatic endothelial cell proliferation fraction; LN: lymph node; NO: lymph node negative; $\mathrm{N}+$ : lymph node positive).

of the University Hospital Antwerp (University of Antwerp, Wilrijk, Belgium). This work was supported by the Fund for Scientific Research Flanders Grant L.3.058.06N. We thank H Elst, Liliane Schelfout and the technical staff of the Laboratory for Pathology of the GH St-Augustinus for expert technical assistance.
Evangelou E, Kyzas P, Trikalinos T (2005) Comparison of the diagnostic accuracy of lymphatic endothelium markers: Bayesian approach. Mod Pathol 18: 1490

Guidi A, Berry D, Broadwater G, Perloff M, Norton L, Barcos MP, Hayes D (2000) Association of angiogenesis in lymph node metastases with outcome of breast cancer. J Natl Cancer Inst 92: 486

Hirakawa S, Kodama S, Kunstfeld R, Kajiya K, Brown LF, Detmar M (2005) VEGF-A induces tumor and sentinel lymph node lymphangiogenesis and promotes lymphatic metastasis. J Exp Med 201: 1089

Kahn H, Marks A (2002) A new monoclonal antibody, D2-40, for detection of lymphatic invasion in primary tumors. Lab Invest 82: 1255 
Koukourakis M, Giatromanolaki A, Sivridis E, Simopoulos C, Gatter K, Harris AL, Jackson D (2005) LYVE-1 immunohistochemical assessment of lymphangiogenesis in endometrial and lung cancer. J Clin Pathol 58: 202

Schacht V, Dadras S, Johnson L, Jackson D, Hong Y, Detmar M (2005) Upregulation of the lymphatic marker podoplanin, a mucin-type transmembrane glycoprotein, in human squamous cell carcinomas and germ cell tumors. Am J Pathol 166: 913

Stessels F, Van Den Eynden G, Van Der Auwera I, Salgado R, Van Den Heuvel E, Harris AL, Jackson DG, Colpaert C, Van Marck EA, Dirix L, Vermeulen P (2004) Breast adenocarcinoma liver metastases, in contrast to colorectal cancer liver metastases, display a non-angiogenic growth pattern that preserves the stroma and lacks hypoxia. Br J Cancer 90: 1429

Straume O, Jackson DG, Akslen LA (2003) Independent prognostic impact of lymphatic vessel density and presence of low-grade lymphangiogenesis in cutaneous melanoma. Clin Cancer Res 9: 250

Van Den Eynden GG, Van Der Auwera I, Van Laere SJ, Colpaert C, Turley H, Harris A, Van Dam P, Dirix L, Vermeulen P, Van Marck EA (2005) Angiogenesis and hypoxia in lymph node metastases is predicted by the angiogenesis and hypoxia in the primary tumour in patients with breast cancer. Br J Cancer 93: 1128

Van Der Auwera I, Van Den Eynden GG, Colpaert C, Van Laere SJ, Van Dam P, Van Marck EA, Dirix L, Vermeulen P (2005) Tumor lymphangiogenesis in inflammatory breast carcinoma: a histomorphometric study. Clin Cancer Res 11: 7637

Vermeulen PB, Gasparini G, Fox SB, Colpaert C, Marson LP, Gion M, Belien JA, de Waal RM, Van Marck E, Magnani E, Weidner N, Harris AL, Dirix LY (2002) Second international consensus on the methodology and criteria of evaluation of angiogenesis quantification in solid human tumours. Eur J Cancer 38(12): 1564 